\title{
Feasibility and utility of facemask sampling in the detection of SARS-CoV-2 during an ongoing pandemic
}

\author{
Dorothy Hui Lin $\mathrm{Ng}^{1}$ C $\cdot$ Mei Yi Sim ${ }^{2}$. Hong Hong Huang ${ }^{2}$. Jean Xiang Ying Sim ${ }^{1}$. Jenny Guek Hong Low ${ }^{1,3}$. \\ Jay Kheng Sit Lim²
}

Received: 12 March 2021 / Accepted: 28 June 2021 / Published online: 5 July 2021

(c) The Author(s), under exclusive licence to Springer-Verlag GmbH Germany, part of Springer Nature 2021

\begin{abstract}
s
Easy access to screening for timely identification and isolation of infectious COVID-19 patients remains crucial in sustaining the international efforts to control COVID-19 spread. A major barrier limiting broad-based screening is the lack of a simple, rapid, and cost-effective COVID-19 testing method. We evaluated the feasibility and utility of facemask sampling in a cohort of 42 COVID-19-positive and 36 COVID-19-negative patients. We used a prototype of Steri-Strips ${ }^{\mathrm{TM}}$ (3 M) applied to the inner surface of looped surgical facemasks (Assure), which was worn by patients for a minimum wear time of $3 \mathrm{~h}$, then removed and sent for SARS-CoV-2 PCR testing. Baseline demographics and symptomatology were also collected. Facemask sampling positivity was highest within the first 5 days of symptomatic presentation. Patients with nasopharyngeal and/or oropharyngeal swab SARS-CoV-2 PCR Ct values $<25.09$ had SARS-CoV-2 detected on facemask sampling, while patients with $\mathrm{Ct}$ values $\geq 25.2$ had no SARS-CoV-2 detected on facemask sampling. Facemask sampling can identify patients with COVID-19 during the early symptomatic phase or those with high viral loads, hence allowing timely identification and isolation of those with the highest transmission risk. Given the widespread use of facemasks, this method can potentially be easily applied to achieve broad-based, or even continuous, population screening.
\end{abstract}

Keywords COVID-19 $\cdot$ SARS-CoV-2 $\cdot$ Rapid diagnostics $\cdot$ Epidemiology

\section{Introduction}

Despite the recent rollout of vaccines, easy access to screening and rapid diagnostics to facilitate early identification and timely isolation of infectious COVID-19 patients remains crucial to control COVID-19 in the ongoing pandemic. To date, nearly 200 million cases of COVID-19 have been confirmed worldwide [1], mostly by nucleic acid amplification testing for SARS-CoV-2 from respiratory samples obtained from the nasopharynx, oropharynx, sputum [2], and, more recently, saliva [3]. The current tally likely represents the tip of the iceberg, as many clinical cases are not tested routinely

Dorothy Hui Lin Ng

huilin.ng@mohh.com.sg

1 Department of Infectious Diseases, Singapore General Hospital, Singapore, Singapore

2 Department of Urology, Singapore General Hospital, Singapore, Singapore

3 Programme in Emerging Infectious Diseases, Duke-NUS Medical School, Singapore, Singapore due to the lack of resources and infrastructural support in many countries.

Nasopharyngeal swabbing (NPS) or oropharyngeal swabbing (OPS) is currently the most commonly used method by healthcare facilities worldwide to diagnose COVID-19. Although regarded as the gold standard diagnostic method, there are significant disadvantages. It is uncomfortable and off-putting and has led to the refusal of testing [4]. Furthermore, it is dependent on trained healthcare workers and availability of personal protective equipment and isolation facilities; therefore, large-scale, well-organized systemic testing programs using NPS/OPS testing are only feasible in some countries, and even then, it is done at staggering costs to healthcare systems and the economy. As a procedure, NPS/OPS swabbing can also cause trauma including minor bleeding to patients and is thus not without risks. Breakpoint fractures of the swab stick with retained foreign bodies $[5,6]$ have been reported, requiring endoscopic or surgical retrieval. The procedure also exposes screening staff to the unnecessary risk of droplet exposure from gag reflexes and coughing. 
SARS-CoV-2 spreads predominantly by respiratory secretions [7, 8], and SARS-CoV-2 can be detected by direct swabbing of the inner surface of facemasks worn by patients with COVID-19 [9]. In the past year, a few studies have evaluated a few methods of facemask sampling (FMS) for the diagnosis of COVID-19. One group tested a prototypic duckbilled surgical mask with specialized 3D-printed sampling strips placed horizontally on the inside of the mask, worn by patients for $30 \mathrm{~min}$, and demonstrated a pick-up rate of $65-70 \%$ in those which were concomitantly NPS positive [10]. Another group used a commercially available gelatine membrane applied on the inner surface of $\mathrm{N} 95$ masks, worn by patients for $30 \mathrm{~min}$, and additionally requested participants to perform certain purposeful vocal tasks. Using this, they demonstrated a pick-up rate of $44.8 \%$ of concomitantly NPSpositive patients [11].

In this study, we investigated the feasibility of facemask sampling (FMS) using a prototype that was based on items that were regularly available in our hospital, such as looped surgical facemasks and wound closure strips, such as Steri-Strips ${ }^{\mathrm{TM}}$ (3 M), as a method for detecting SARSCoV-2. The yield of FMS was compared with NPS/OPS using the SARS-CoV-2 reverse transcription quantitative PCR (RT qPCR) method.

\section{Materials and methods}

\section{Patient recruitment and facemask sampling}

Patients diagnosed with COVID-19 with positive NPS/ OPSs were admitted to our institution and placed in isolation within 1-2 days after confirmation of a positive SARSCoV-2 PCR test result. They were housed individually in negative-pressure isolation rooms with airborne precautions. Patients were recruited within $72 \mathrm{~h}$ of their first positive NPS/OPS. As negative controls, patients who were admitted to hospital via the emergency department who presented with fever or acute respiratory infection (ARI) symptoms or fulfilled Ministry of Health, Singapore (MOH) isolation criteria [12], and who had at least two negative NPS or OPS performed $24 \mathrm{~h}$ apart, were also recruited. All participants were instructed to don looped surgical facemasks (Assure) with three Steri-Strips ${ }^{\mathrm{TM}}(3 \mathrm{M})$ stuck horizontally on the inner surface of the facemask (Fig. 1) for a minimum of $3 \mathrm{~h}$ of wear time. There were no restrictions on vocal maneuvers, and patients could cough, sneeze, talk, laugh, or sleep as desired. The Steri-Strips ${ }^{\mathrm{TM}}$ were then removed from the facemask by personnel dressed in full personal protective equipment (including N95 masks, face shields, hair net, yellow gown, and gloves) using sterile forceps, applied onto a sterile pipette trip with no direct handling of the strips, and deposited directly into vials containing viral transport media for stabilization and transported at room temperature with
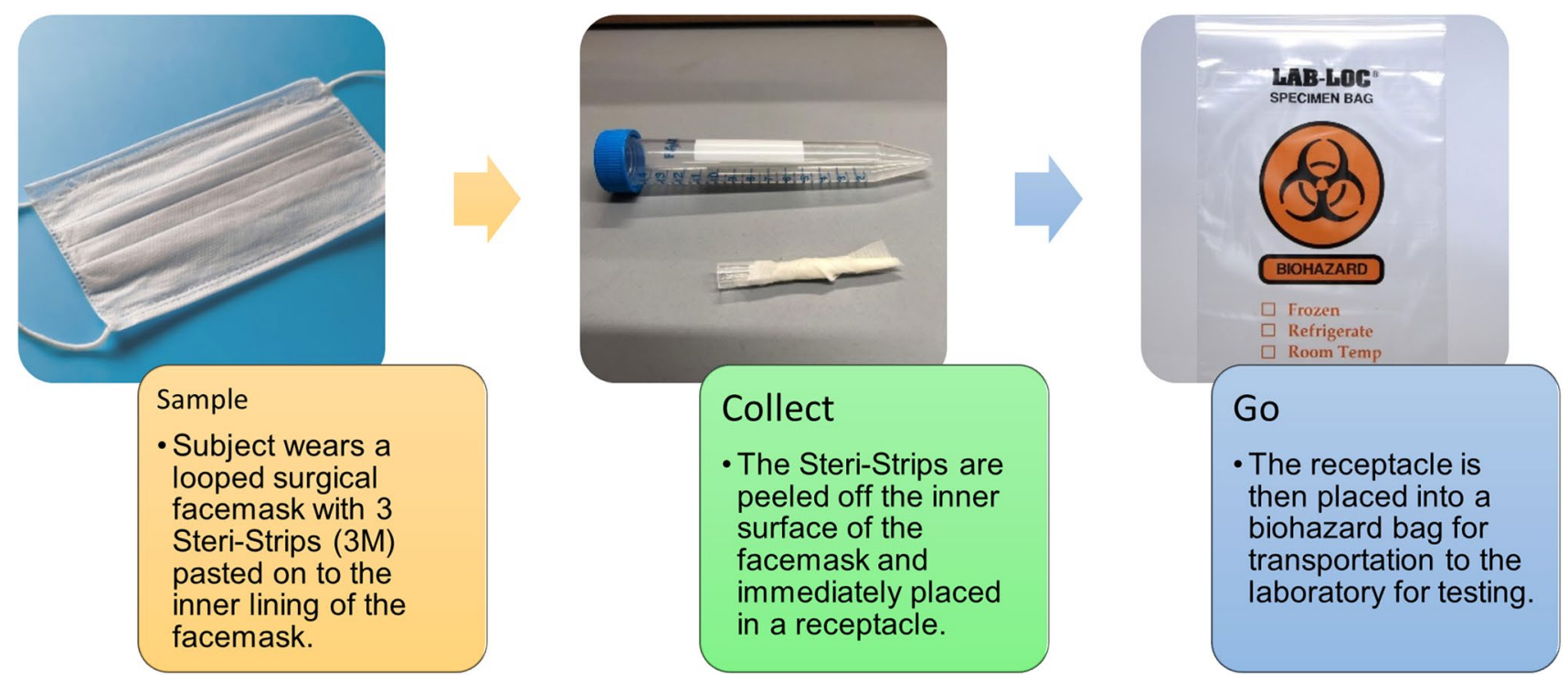

Fig. 1 Schemata of the FMS process. Patients were asked to don standard looped surgical face masks (Assure) with three SteriStrips $^{\mathrm{TM}}(3 \mathrm{M})$ applied horizontally to the inner surface of the mask. After at least $3 \mathrm{~h}$ of minimum wear time, the strips were removed with sterile forceps and placed into a vial containing transport media, and then into appropriate biohazard packaging for delivery to the laboratory for testing 
Biological Agents and Toxins Act (Transportation) Regulation approved precautions.

\section{SARS-CoV-2 RT-PCR}

SARS-CoV-2 testing for this study was carried out by two College of American Pathologists (CAP)-accredited and Ministry of Health, Singapore-licensed service laboratories (Lucence Diagnostics Pte Ltd. and Singapore General Hospital Molecular Pathology Laboratory). For Lucence Diagnostics Pte Ltd., samples were placed in a primary collection tube which contained $1 \mathrm{ml}$ of proprietary transport media. Viral nucleic acid was extracted using Viral Nucleic Acid Extraction Kit II (Geneaid Biotech Ltd, Taiwan) or QIAsymphony DSP Virus/Pathogen Kit (Qiagen, Germany) according to manufacturer's instructions. For samples tested in the Singapore General Hospital Molecular Pathology Laboratory, samples were placed in a primary collection tube which contained $1 \mathrm{ml}$ of laboratory proprietary lysis reagent. Viral nucleic acid extraction and SARS-CoV-2 diagnosis were carried out using the cobas SARS-CoV-2 test (Roche) according to the manufacturer's instructions. The SARS$\mathrm{CoV}-2$ diagnosis was carried out using the CDC 2019-nCoV real-time (RT)-PCR diagnostic panel (USA) which targets the nucleocapsid gene of the virus [13]. Samples with a reported $\mathrm{Ct}$ value $<40$ were considered positive.

\section{Ethics approval}

Ethics (CRIB reference number: 2020/2343) and biosafety approvals were obtained for this study. All patients gave informed consent to the study.

\section{Statistical analyses}

Statistical analyses were conducted using SPSS statistics (IBM, v26.0) and GraphPad Prism software. Pearson chi-square and Fisher's exact tests were used for categorical variable outcomes. Mann-Whitney $U$-test was used for nonparametric variables. Tables were made using Microsoft Excel software. Figure graphics were generated using GraphPad Prism software.

\section{Results}

\section{Patient demographics and characteristics}

Between June 24, 2020 and November 2, 2020, a total of 90 patients were screened and recruited from the isolation wards at the Singapore General Hospital. Of these, 78 agreed to participate in the study and completed the sample collection described previously (Fig. 1). Fortytwo patients with SARS-CoV-2 detected via NPS/OPS and 36 control patients with at least two negative NPS/ OPS were recruited. Baseline demographics, including age, ethnicity, and past medical history, were comparable between the positive and control groups (Table 1). The median duration of symptomatic illness prior to FMS was 4 days for NPS-/OPS-positive patients and 5 days for NPS-/OPS-negative patients $(p=0.276)$. More patients in the NPS-/OPS-positive cohort presented with a sore throat $(23.8 \%$ vs $5.6 \%, p=0.031$, and more patients in the NPS-/OPS-negative cohort presented with fever (55.6\% vs $31 \%, p=0.039$ ); otherwise, there was no significant difference in presenting symptoms. Around $35.7 \%$ of the NPS-/OPS-positive patients were asymptomatic, while $5.4 \%$ of NPS-/OPS-negative patients were asymptomatic $(p=0.002)$ (Table 2). All patients were hemodynamically stable at recruitment, and none required supplemental oxygen throughout hospitalization.
Table 1 A comparison of the demographics of patients with COVID-19 and negative controls

\begin{tabular}{llll}
\hline Feature & \multicolumn{2}{l}{ NPS/OPS } & $p$ value \\
\cline { 2 - 3 } & Positive, $n=42$ & Negative, $n=36$ & \\
\hline Age (years), median (IQR) & $47.5(6.0)$ & $45.0(24.0)$ & 0.976 \\
Duration of illness before FMS, median (IQR) & $4.0(2.0)$ & $5.0(9.0)$ & 0.276 \\
Number of days between NPS/OPS and FMS, & $3.0(1.0)$ & $1.0(2.0)$ & $<0.001$ \\
median (IQR) & & & \\
Ethnicity, $n(\%)$ & & & 0.072 \\
Bangladeshi & $23(54.8)$ & $11(30.6)$ & \\
Chinese & $9(21.4)$ & $11(30.6)$ & \\
$\quad$ Malay & $0(0.0)$ & $3(8.3)$ & \\
Indian & $8(19.0)$ & $6(16.7)$ & $5(13.0)$ \\
$\quad$ Others & $2(4.8)$ & & \\
\hline
\end{tabular}

Mann-Whitney $U$-test for continuous variables and Fisher's exact test for categorical variables 
Table 2 A comparison of the symptomology of patients with COVID-19 and negative controls

\begin{tabular}{|c|c|c|c|}
\hline \multirow[t]{2}{*}{ Feature } & \multicolumn{2}{|l|}{ NPS/OPS, $n(\%)$} & \multirow[t]{2}{*}{$p$ value } \\
\hline & Positive, $n=42$ & Negative, $n=36$ & \\
\hline \multicolumn{4}{|l|}{ Past medical history } \\
\hline Nil & $29(69.0)$ & $18(50.0)$ & 0.17 \\
\hline Hypertension & $5(11.9)$ & $7(19.4)$ & 0.531 \\
\hline Hyperlipidaemia & $0(0.0)$ & $6(16.7)$ & 0.008 \\
\hline Diabetes mellitus & $2(4.8)$ & $5(13.9)$ & 0.239 \\
\hline $\begin{array}{l}\text { Pre-existing upper } \\
\text { respiratory tract } \\
\text { conditions }\end{array}$ & $3(7.1)$ & $5(13.9)$ & 0.460 \\
\hline $\begin{array}{l}\text { Pre-existing lung } \\
\text { conditions }\end{array}$ & $1(2.4)$ & $2(5.6)$ & 0.593 \\
\hline $\begin{array}{l}\text { Cerebrovascular } \\
\text { accident }\end{array}$ & $0(0.0)$ & $2(5.6)$ & 0.210 \\
\hline Obesity & $0(0.0)$ & $2(5.6)$ & 0.210 \\
\hline Thyroid conditions & $0(0.0)$ & $2(5.6)$ & 0.210 \\
\hline $\begin{array}{l}\text { Single organ trans- } \\
\text { plant }\end{array}$ & $1(2.4)$ & $0(0.0)$ & 1.000 \\
\hline Others & $1(2.4)$ & $8(22.2)$ & 0.010 \\
\hline Asymptomatic & $15(35.7)$ & $2(5.4)$ & 0.002 \\
\hline Symptom & $27(64.3)$ & $34(94.4)$ & \\
\hline Cough & $15(35.7)$ & $9(25.0)$ & 0.336 \\
\hline Fever & $13(31.0)$ & $20(55.6)$ & 0.039 \\
\hline Sore throat & $10(23.8)$ & $2(5.6)$ & 0.031 \\
\hline Runny nose & $7(16.7)$ & $2(5.6)$ & 0.166 \\
\hline Abdominal pain & $2(4.8)$ & $2(5.6)$ & 1.000 \\
\hline Myalgia & $2(4.8)$ & $4(11.1)$ & 0.406 \\
\hline Ansomia & $3(7.1)$ & $0(0.0)$ & 0.245 \\
\hline Aguesia & $3(7.1)$ & $2(5.6)$ & 1.000 \\
\hline Diarrhea & $2(4.8)$ & $0(0.0)$ & 0.497 \\
\hline Breathlessness & $3(7.1)$ & $6(16.7)$ & 0.288 \\
\hline Blocked nose & $1(2.4)$ & $0(0.0)$ & 1.000 \\
\hline Chest discomfort & $1(2.4)$ & $3(8.3)$ & 0.330 \\
\hline Nausea & $0(0.0)$ & $1(2.8)$ & 0.462 \\
\hline
\end{tabular}

Fisher's exact test for categorical variables

\section{Facemask sampling positivity is highest within 5 days of onset of symptoms}

In the cohort of 42 NPS-/OPS-positive patients, a total of 16 FMS strips were positive by SARS-CoV-2 PCR testing $(38.1 \%)$, while all 36 patients in the NPS-/OPS-negative cohort had negative FMS results. The positive predictive value and negative predictive value of FMS were $100 \%$ and $58.1 \%(52.2-63.7 \%)$, respectively. The overall sensitivity and specificity of FMS were $38.1 \%$ (23.6-54.4\%) and $100 \%$ (90.3-100\%), respectively. The overall accuracy was $66.7 \%$.

In NPS-/OPS-positive patients, FMS positivity was significantly associated with time from onset of fever (Fisher's exact test, $p=0.0291$ ) (Fig. 2a) or fever and ARI (FARI) symptoms (Fisher's exact test, $p=0.0476$ ) (Fig. 2b). FMS was positive in $6 / 7$ (85.7\%) of patients tested within the first 5 days of presentation with fever or FARI symptoms (Fig. 2a, b). In patients who presented with ARI symptoms without fever, FMS was positive in 10/16 (62.5\%) of patients tested within the first 5 days, but overall, there did not seem to be a significant association with FMS positivity and time from onset of ARI symptoms (Fig. 2b).

\section{Facemask sampling positivity correlates with lower NPS/OPS Ct values}

NPS/OPS Ct values were available for $36 / 42$ patients with COVID-19. Of these 36 patients, those who had corresponding positive FMS had significantly lower NPS/OPS $\mathrm{Ct}$ values. All patients with NPS/OPS Ct values $<25.09$ had SARS-CoV-2 detected by FMS, while patients with NPS/ OPS Ct values $\geq 25.2$ did not have SARS-CoV-2 detected on FMS (Pearson's $r=-0.89, p<0.0001$ ) (Fig. 3a). Symptomatic patients were more likely to have lower NPS/ OPS Ct values than asymptomatic patients (mean values 24.95 vs $34.62, p=0.027$ ) (Fig. $3 b$ ). The mean $\mathrm{Ct}$ values on FMS strips were 29.4 (20.51-35.77) for N1 and 30.19 (24.3-35.29) for N2 (data not shown). There was no correlation between FMS Ct values and NPS/OPS Ct values (data not shown).
Fig. 2 FMS positivity is highest within 5 days of onset of symptoms. Scatter dot plots showing days from onset of a fever, $\mathbf{b}$ fever and ARI (FARI), or c ARI symptoms on which FMS was performed. The blackfilled circles (O) represent FMS results from individual patients. The solid line represents day 5 . * indicates $p<0.05$
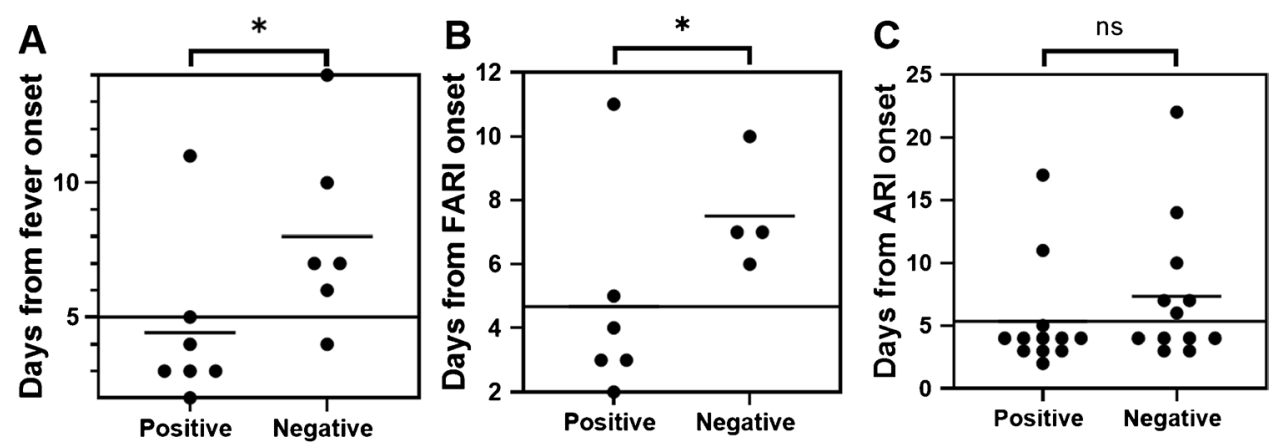


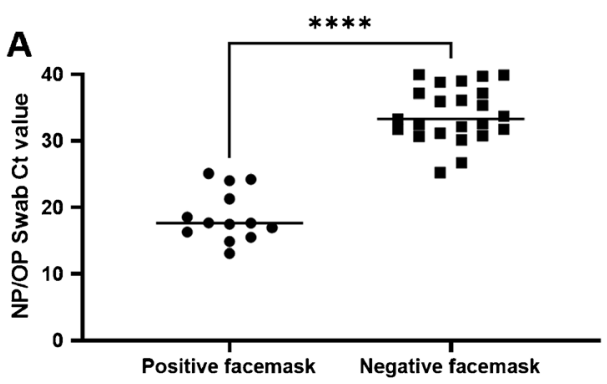

Fig. 3 FMS positivity correlates with lower NPS/OPS Ct values. a Scatter dot plot showing NPS/OPS swab Ct values in 36 patients with positive or negative FMS. The black-filled circles () represent individual patients with detectable SARS-CoV-2 by PCR on FMS. The black squares $(\mathbf{\square})$ represent individual patients with undetectable SARS-CoV-2 by PCR on FMS. b Scatter dot plot showing NPS/

\section{Discussion}

As the COVID-19 pandemic matures towards "the new normal" with anticipated repeated waves of infection [14], a cost-effective and reproducible method for SARS-CoV-2 testing, which allows for self-collection or rapid on-site collection for group screening, is becoming crucial for sustaining worldwide efforts in maintaining testing rigor even with rapid vaccine rollouts.

In this study, our purpose was to assess the utility and feasibility of FMS using materials which were already widely available, such as wound closure strips and surgical facemasks (Table 3). Using this method, FMS had a pick-up rate of $62.6-87.5 \%$ of patients who were tested within the first 5 days of symptom onset, which is consistent with previous studies [10,11]. Additionally, FMS was able to distinguish symptomatic patients with COVID-19 from those presenting with fever or ARI symptoms without COVID-19. The reason why the yield of FMS would

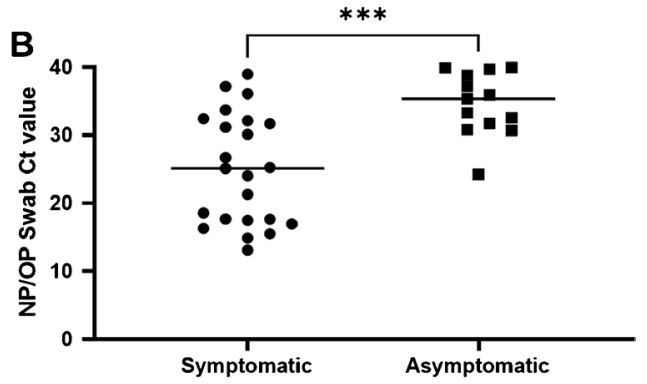

OPS swab Ct values in 36 patients who were symptomatic vs asymptomatic at presentation. The black-filled circles $(\mathbf{O})$ represent individual COVID-19 patients presenting with fever and/or ARI symptoms. The black squares $(\mathbf{\square})$ represent individual COVID-19 patients who presented asymptomatically. $* * *$ indicates $p<0.001$. $* * * *$ indicates $p<0.0001$

be less in those presenting with ARI symptoms alone without fever is unclear, but we postulate that it may have been affected by the subjectivity of reported symptoms, whereas fever may have been a more objective measurement.

We also observed that an NPS/OPS Ct value $<25.09$ was the value upon which FMS would turn positive by PCR. This tight correlation between NPS/OPS Ct values and detection on FMS remained consistent even though the samples had been sent to six different laboratories and processed with different protocols and reagents. In asymptomatic patients, FMS only picked up asymptomatic patients with NPS/OPS swab Ct value $<24$. However, the sensitivity and specificity of FMS in asymptomatic patients were limited in this study, as most of our asymptomatic patients had NPS/OPS swab Ct values $>24$.

$\mathrm{Ct}$ values have been used as a surrogate marker for "viral load," with a low Ct value indicating a high viral RNA amount, and vice versa. Because SARS-CoV-2 can remain detectable in NPS/OPS swabs for up to 5 weeks after onset of symptoms, even though patients are no longer infectious

Table 3 Study costs and operational considerations for facemask sampling

\begin{tabular}{|c|c|c|}
\hline Item & Cost/test (Singapore dollar) & Other considerations \\
\hline 1. Surgical looped facemask (Assure) & $\$ 0.13$ & \multirow{2}{*}{$\begin{array}{l}\text { Any facemask, even reusable facemasks which have been } \\
\text { washed and dried, can potentially be used for sampling } \\
\text { with strips. On the other hand, swabbing the inner sur- } \\
\text { face of the facemask may result in variability depending } \\
\text { on the mask type and method of swabbing }\end{array}$} \\
\hline 2. Steri-Strips $(3 \mathrm{M})$ & $\$ 1.20$ & \\
\hline 3. Disposable sterile forceps & $\$ 2.50$ & \multirow[t]{3}{*}{ Wound closure strips are widely available } \\
\hline $\begin{array}{l}\text { 4. Equipment and reagents for sample collection and } \\
\text { SARS-CoV-2 RT-PCR }\end{array}$ & \multirow[t]{2}{*}{ As per laboratory } & \\
\hline Operational considerations & & \\
\hline \multicolumn{3}{|c|}{$\begin{array}{l}\text { 1. Minimal training needed to perform sample collection, and the use of sterile forceps enables it to be done in an aseptic manner to reduce } \\
\text { contamination }\end{array}$} \\
\hline \multicolumn{3}{|c|}{ 2. Facemask sampling with removable strips can be operationalized into batch testing } \\
\hline
\end{tabular}


[15], not having the means to differentiate infectious patients from those who have limited or no transmission risk has meant that many swab-positive patients may have been isolated unnecessarily and kept in quarantine over prolonged periods $[16,17]$. Therefore, there has been an increasing debate regarding defining $\mathrm{Ct}$ thresholds or cutoffs to differentiate infectious patients from noninfectious or recovering COVID-19 patients to guide isolation or deisolation policies. Several studies have attempted to evaluate the correlation between NPS/OPS swab $\mathrm{Ct}$ values and SARS-CoV-2 recovery in cell culture. In a multicenter cohort of 73 COVID19 patients in Singapore, when the Ct value was $\geq 30$, no virus could be isolated [18]. Another report suggested that a Ct value $>33-34$ was not associated with cell culture viral recovery [19], and another showed that only those with $\mathrm{Ct}$ mean average values of $18.8 \pm 3.4$ and median of 18.17 had virus recovery in cell culture [20]. Cell culture infectivity was no longer observed when $\mathrm{Ct}$ values were above 24 and after 8 days from symptom onset in one study [21]. Two virus culture studies found that no infectious isolates were obtained from any sample taken after day 8 of symptoms despite ongoing high viral loads $[19,22]$. Although only the minority of patients with higher $\mathrm{Ct}$ values have a cultivable virus, the issues of operator-dependent variability, the lack of quantitative assays, and the lack of standardization across tests make it challenging for a consensus on $\mathrm{Ct}$ value thresholds and infectivity to be reached.

Although the sensitivity of FMS is lower than the reported figures for midturbinate, anterior nasal, combined NPS/OPS, or saliva as compared to NPS alone [23], it has the advantages of low cost, accessibility, and noninvasiveness, and, if combined with rapid antigen detection technologies, may potentially have rapid sample-to-diagnosis time - and hence can be of high cost-effective epidemiological value in settings where broad, regular, mass screening is required [16, 24]. With increasing cultural adaptation to mask wearing, widespread utilization of FMS can potentially be easy to execute to allow frequent testing — even up to multiple times per week. As FMS is highly sensitive at the early stage of illness, even potentially in asymptomatic patients, and in patients with low Ct values, a strategy of frequent mass surveillance can potentially identify those at the beginning of infection for timely isolation to stop onward spread, while dovetailing efforts for more detailed testing and quarantine if necessary.

\section{Limitations}

There are several operational limitations of this study which need to be addressed.

Firstly, it is a single-center study with small sample size. Although we had initially aimed to recruit at least
200 patients, the drastic decrease of COVID-19 cases in Singapore after July 2020 curtailed our recruitment.

Secondly, a portion of our positive cohort was asymptomatic and was incidentally discovered through routine biweekly NPS/OPS screening of high-risk groups which were being conducted at the time. For these asymptomatic patients, it was not possible to ascertain the duration of infection - and their high NPS/OPS Ct values suggested that most of them may have been at the recovery or noninfectious phase of illness.

Thirdly, due to operational workflows in our institution at the time, FMS was not done on the same day as NPS/ OPS swabbing and swabs were performed by different healthcare workers and processed by different laboratories. Nevertheless, healthcare workers in our institution had undergone training on a single NPS/OPS collection protocol, which would decrease the heterogeneity of the reported NPS/OPS Ct values.

Fourthly, we are unable to definitively exclude the possibility of facemask strip contamination by external droplets or biological fluids. Indeed, extensive environmental contamination by SARS-CoV-2 in the environments of infected patients has been demonstrated in multiple studies in healthcare and community settings [9, 25-27]. Nevertheless, in our study protocol, the study facemasks were worn for specific periods of time by patients singly housed in negative-pressure isolation rooms and who were only visited by staff in full personal protective equipment. Sterile single-use forceps were used by trained personnel to transfer the wound closure strips immediately from the facemask directly into transportation vials. Therefore, external contamination of the facemask strips would have been highly unlikely.

In real-life situations, however, external contamination during self-collection of the strips can result in confounding false-positive results. Potential avenues to mitigate this include the use of rapid antigen detection technologies that bypass the steps needed for sample processing for RT-PCR. Conversely, empty or inadequately worn masks can result in false-negative facemask strips. We speculate that the addition of accessory detectors such as end-tidal carbon dioxide detector strips, which can semiquantitatively measure the amount of exhaled gases by virtue of carbon dioxide detection, or starch strips which changes color on contact with saliva, can serve as proxies for oropharyngeal or nasopharyngeal exposure - these also have the benefit of being potentially adaptable for rapid point-of-care facemask sampling. Alternatively, further studies could be done to see if introducing vocal maneuver, such as purposeful open-mouth breathing or coughing, would help to increase the sensitivity. False-negative results due to the presence of inhibitors of PCR amplification are unlikely due to the use of internal extraction and amplification controls. 


\section{Conclusions}

As pandemic efforts mature and pivot towards strategies that allow for the "return to normalcy," new methods for sustainable and broad-based screening are crucial for the gradual reopening of societies. FMS using widely available materials can offer the potential for easy screening of febrile patients during the early infectious phase of illness and thus facilitate timely isolation decisions.

Acknowledgements The authors would like to thank Dr. Tan Min-Han and the team from Lucence; A/Prof. Chiminda Jayampath Seneviratne from the National Dental Centre Singapore; and Dr. Chan Kian Sing, Dr. Kenneth Goh, and Dr. Shireen Tan from the Department of Microbiology, SGH, for their helpful comments and critical appraisal of this work. The authors would like to acknowledge and thank all healthcare colleagues globally for their unstinting fight against COVID-19.

Funding Singapore General Hospital COVID-19 Resilience Innovation Grant. Low J.G.H. is funded by the National Medical Research Council, Singapore, through the Clinician Scientist Award.

Data availability Nil.

Code availability Nil.

\section{Declarations}

Ethics approval Ethics (CRIB reference number: 2020/2343) and biosafety approvals were obtained for this study.

Consent to participate All patients gave verbal informed consent to the study.

Consent for publication Not applicable.

Conflict of interest The authors declare no competing interests.

\section{References}

1. WHO (2020) Coronavirus disease (COVID-2019) situation reports, https://www.who.int/emergencies/diseases/novel-coron avirus-2019/situation-reports/]. Cited July 22, 2020

2. Wang W, Xu Y, Gao R, Lu R, Han K, Wu G, Tan W (2020) Detection of SARS-CoV-2 in different types of clinical specimens. Jama 323(18): 1843-1844

3. Wyllie AL, Fournier J, Casanovas-Massana A, Campbell M, Tokuyama M, Vijayakumar P, Warren JL, Geng B, Muenker MC, Moore AJ (2020) Saliva or nasopharyngeal swab specimens for detection of SARS-CoV-2. N Engl J Med 383(13):1283-1286

4. McDermott JH, Newman WG (2020) Refusal of viral testing during the SARS-CoV-2 pandemic. Clin Med 20(5):e163

5. Mughal Z, Luff E, Okonkwo O, Hall C (2020) Test, test, test-a complication of testing for coronavirus disease 2019 with nasal swabs. J Laryngol Otol 134(7):646-649

6. Hussein MS, Hameed M, Allangawi M, Abdelsattar H, Haq IU (2020) An unusual and unexpected result of diagnostic sampling for COVID-19. Cureus 12(10)
7. Meyerowitz EA, Richterman A, Gandhi RT, Sax PE (2021) Transmission of SARS-CoV-2: a review of viral, host, and environmental factors. Ann Intern Med 174(1):69-79

8. Sommerstein R, Fux CA, Vuichard-Gysin D, Abbas M, Marschall J, Balmelli C, Troillet N, Harbarth S, Schlegel M, Widmer A (2020) Risk of SARS-CoV-2 transmission by aerosols, the rational use of masks, and protection of healthcare workers from COVID-19. Antimicrob Resist Infect Control 9(1):1-8

9. Dargahi A, Jeddi F, Ghobadi H, Vosoughi M, Karami C, Sarailoo M, Hadisi A, Mokhtari SA, Haghighi SB, Sadeghi H (2021) Evaluation of masks' internal and external surfaces used by health care workers and patients in coronavirus-2 (SARSCoV-2) wards. Environ Res 196:110948

10. Williams CM, Pan D, Decker J, Wisniewska A, Fletcher E, Sze S, Assadi S, Haigh R, Abdulwhhab M, Bird P (2021) Exhaled SARS-CoV-2 quantified by face-mask sampling in hospitalised patients with COVID-19. J Infect 82(6):253-259

11. Sriraman K, Shaikh A, Parikh S, Udupa S, Chatterjee N, Shastri J, Mistry N (2021) Non-invasive adapted N-95 mask sampling captures variation in viral particles expelled by COVID-19 patients: implications in understanding SARS-CoV2 transmission. PloS one 16(4):e0249525

12. MOH updates on COVID-19 (coronavirus disease 2019) local situation, https://www.moh.gov.sg/covid-19. Cited February 22 2021

13. CDC (2020) Research use only 2019-novel coronavirus (2019$\mathrm{nCoV}$ ) real-time RT-PCR primers and probes, https://www.cdc. gov/coronavirus/2019-ncov/lab/rt-pcr-panel-primer-probes. html]

14. Chandra S, Christensen J, Chandra M, Paneth N (2021) Pandemic reemergence and four waves of excess mortality coinciding with the 1918 influenza pandemic in Michigan: insights for COVID-19. Am J Publ Health 111(3):430-437

15. Xiao AT, Tong YX, Zhang S (2020) Profile of RT-PCR for SARSCoV-2: a preliminary study from 56 COVID-19 patients. Clin Infect Dis

16. Mina MJ, Parker R, Larremore DB (2020) Rethinking COVID19 test sensitivity - a strategy for containment. N Engl J Med 383(22):e120

17. Tom MR, Mina MJ (2020) To interpret the SARS-CoV-2 test, consider the cycle threshold value. Clin Infect Dis 71(16):2252-2254

18. Young BE, Ong SW, Ng LF, Anderson DE, Chia WN, Chia PY, Ang LW, Mak T-M, Kalimuddin S, Chai LYA (2020) Viral dynamics and immune correlates of COVID-19 disease severity. Clin Infect Dis:ciaa1280

19. La Scola B, Le Bideau M, Andreani J, Hoang VT, Grimaldier C, Colson P, Gautret P, Raoult D (2020) Viral RNA load as determined by cell culture as a management tool for discharge of SARS-CoV-2 patients from infectious disease wards. Eur J Clin Microbiol Infect Dis 39(6):1059

20. Gniazdowski V, Morris CP, Wohl S, Mehoke T, Ramakrishnan S, Thielen P, Powell H, Smith B, Armstrong DT, Herrera M (2020) Repeat COVID-19 molecular testing: correlation of SARS-CoV-2 culture with molecular assays and cycle thresholds. Clin Infect Dis:ciaa1616

21. Bullard J, Dust K, Funk D, Strong JE, Alexander D, Garnett L, Boodman C, Bello A, Hedley A, Schiffman Z (2020) Predicting infectious severe acute respiratory syndrome coronavirus 2 from diagnostic samples. Clin Infect Dis 71(10):2663-2666

22. Wölfel R, Corman VM, Guggemos W, Seilmaier M, Zange S, Müller MA, Niemeyer D, Jones TC, Vollmar P, Rothe C (2020) Virological assessment of hospitalized patients with COVID2019. Nature 581(7809):465-469

23. Hanson K, Caliendo A, Arias C (2020) IDSA guidelines on the diagnosis of covid-19: molecular diagnostic testing. Clin Infect Dis:ciaa1343 
24. Larremore DB, Wilder B, Lester E, Shehata S, Burke JM, Hay JA, Tambe M, Mina MJ, Parker R (2021) Test sensitivity is secondary to frequency and turnaround time for COVID-19 screening. Sci Adv 7(1):eabd5393

25. Ong SWX, Tan YK, Chia PY, Lee TH, Ng OT, Wong MSY, Marimuthu K (2020) Air, surface environmental, and personal protective equipment contamination by severe acute respiratory syndrome coronavirus 2 (SARS-CoV-2) from a symptomatic patient. Jama 323(16):1610-1612

26. Chia PY, Coleman KK, Tan YK, Ong SWX, Gum M, Lau SK, Lim XF, Lim AS, Sutjipto S, Lee PH (2020) Detection of air and surface contamination by SARS-CoV-2 in hospital rooms of infected patients. Nat Commun 11(1):1-7
27. Piana A, Colucci ME, Valeriani F, Marcolongo A, Sotgiu G, Pasquarella C, Margarucci LM, Petrucca A, Gianfranceschi G, Babudieri S (2021) Monitoring COVID-19 transmission risks by quantitative real-time PCR tracing of droplets in hospital and living environments. MSphere 6(1):e01070-01020

Publisher's note Springer Nature remains neutral with regard to jurisdictional claims in published maps and institutional affiliations. 